\title{
Correction to: Late toxicity of image-guided hypofractionated radiotherapy for prostate: non-randomized comparison with conventional fractionation
}

\author{
Barbara Alicja Jereczek-Fossa ${ }^{1,2} \cdot$ Alessia Surgo $^{2}\left({ }^{0} \cdot\right.$ Patrick Maisonneuve $^{3} \cdot$ Andrea Maucieri $^{2}$. \\ Marianna Alessandra Gerardi ${ }^{2}$. Dario Zerini ${ }^{2}$. Giulia Marvaso ${ }^{2} \cdot$ Delia Ciardo $^{2} \cdot$ Stefania Volpe $^{1,2}$. \\ Damaris Patricia Rojas ${ }^{1,2}$. Giulia Riva ${ }^{1,2}$. Ombretta Alessandro ${ }^{1,2}$. Samantha Dicuonzo ${ }^{2}$. Giuseppe Fanetti ${ }^{1,2}$. \\ Paola Romanelli ${ }^{2}$. Anna Starzyńska ${ }^{4}$. Federica Cattani ${ }^{5} \cdot$ Raffaella Cambria $^{5}$. Cristiana Fodor ${ }^{2}$. Cristina Garibaldi ${ }^{5}$. \\ Chiara Romanò ${ }^{5,6} \cdot$ Ottavio De Cobelli $^{1,7} \cdot$ Roberto Orecchia $^{8}$
}

Published online: 8 October 2019

(c) Italian Society of Medical Radiology 2019

\section{Correction to: La radiologia medica (2019) 124:65-78 https://doi.org/10.1007/s11547-018-0937-9}

Unfortunately, the denomination of the IEO was incorrectly published in the affiliation of this original article. The complete correct denomination of IEO should read as follows:

IEO, European Institute of Oncology IRCCS, Milan.
Publisher's Note Springer Nature remains neutral with regard to jurisdictional claims in published maps and institutional affiliations.

The original article can be found online at https://doi.org/10.1007/ s11547-018-0937-9.

Alessia Surgo

surgo.alessia@ieo.it

1 Department of Oncology and Hemato-Oncology, University of Milan, Milan, Italy

2 Department of Radiotherapy, IEO, European Institute of Oncology IRCCS, Via Ripamonti 435, 20141 Milan, Italy

3 Division of Epidemiology and Biostatistics, IEO, European Institute of Oncology IRCCS, Milan, Italy

4 Department of Oral Surgery, Medical University of Gdańsk, Gdańsk, Poland

5 Department of Medical Physics, IEO, European Institute of Oncology IRCCS, Milan, Italy

6 Department of Physics, University of Milan, Milan, Italy

7 Department of Urology, IEO, European Institute of Oncology IRCCS, Milan, Italy

8 Scientific Directorate, IEO, European Institute of Oncology IRCCS, Milan, Italy 